\title{
Single short-chain conjugated polymer studied with optical spectroscopy: A donor-accepter system
}

\author{
T.-S. Lim, ${ }^{1}$ J.-C. Hsiang, ${ }^{2}$ J. D. White, ${ }^{3}$ J. H. Hsu, ${ }^{4}$ Y. L. Fan, ${ }^{5}$ K. F. Lin,${ }^{5}$ and W. S. Fann ${ }^{1,2}$ \\ ${ }^{1}$ Institute of Atomic and Molecular Sciences, Academia Sinica, P.O. Box 23-116, Taipei, Taiwan, Republic of China \\ ${ }^{2}$ Department of Physics and Institute of Polymer Science and Engineering, National Taiwan University, Taipei, Taiwan, \\ Republic of China \\ ${ }^{3}$ Department of Electrical Engineering, Yuan Ze University, Chungli, Taiwan, Republic of China \\ ${ }^{4}$ Department of Material Science and Optoelectronic Engineering, National Sun Yat-sen University, Kaohsiung, Taiwan, \\ Republic of China \\ ${ }^{5}$ Department of Materials Science and Engineering, National Taiwan University, Taipei, Taiwan, Republic of China
}

(Received 31 October 2006; revised manuscript received 15 February 2007; published 18 April 2007)

Single rodlike poly[2-methoxy, 5-(2'-ethyl-hexyloxy)-p-phenylene-vinylene] (MEH-PPV), in which the chain length supports only two or three chromophores, was studied using optical spectroscopy in order to elucidate exciton dynamics within a single conjugated polymer. Emission at one to three discrete intensity levels coupled with on/off blinking was seen in the fluorescence time traces. Drops in emission intensity were accompanied in the majority of cases by an abrupt spectral blueshift and a corresponding increase in the fluorescence lifetime. Photon correlation measurements indicated that at any point in time there was only one emitter active. This suggests that the two or three chromophores in a short-chain polymer act as a typical donor-acceptor system with energy absorbed by the donors (shorter chromophores) transferring to the acceptor (longer chromophore) along the polymer backbone before emission. Once the acceptor is quenched, a donor takes turns to emit.

DOI: 10.1103/PhysRevB.75.165204

\section{INTRODUCTION}

Unique electronic and optical properties ${ }^{1}$ coupled with their ease of processing, make luminescent conjugated polymers attractive for a variety of applications such as plastic light-emitting diodes and solar cells. ${ }^{2}$ While these highly conjugated linear macromolecules exhibit certain semiconductorlike properties, their electronic properties are much more complex than their inorganic analog. Even the nature of excitations, the most common of which is called an exciton, are complicated. ${ }^{3}$ Basically, two kinds of excitons have been identified. ${ }^{3-6}$ In the first, extended $\pi$ conjugation along sections of the polymer backbone support intrachain excitons. In the second, coupling of segments through space of two adjacent chain segments support interchain excitons. This coupling can either be between chains near to each other in solid film or between segments on a folded single chain. The optical properties of interchain excitons are even more complicated then the intrachain excitons, depending strongly on the aggregation state of polymers. In this paper, the majority of our discussion focuses on the intrachain exciton-as our polymer's conformation inhibits the formation of interchain excitons.

Within a single extended polymer interrupted conjugation-for example due to single bonds substituting double bonds or chain bending/twisting forced by the surroundings-results in the polymer being divided into a number of segments, called chromophores, each of which has the potential to absorb and emit light. The distribution of the conjugation lengths of these chromophores is reflected in the broad absorption spectrum of polymers in solution. ${ }^{7}$ Once formed, excitons migrate from short to longer conjugated segments resulting in a photoluminescence (PL) spectrum that is narrower than the absorption spectrum. The na-
PACS number(s): 78.55. $-\mathrm{m}, 34.30 .+\mathrm{h}, 78.30 . \mathrm{Jw}, 42.50 . \mathrm{Ar}$

ture of this migration is a fundamental problem in luminescent conjugated polymer physics. Much attention has been given to the relation of the exciton migration within a conjugated polymer to its conformation. ${ }^{8-11}$ Experimental and theoretical results demonstrate that energy transfer (ET) or exciton migration along the polymer backbone is less efficient than three-dimensional intrachain ET. ${ }^{12-14}$ (For the purposes of this paper, ET refers solely to events occurring on the picosecond to nanosecond time scale. Processes occurring on the femtosecond time scale are beyond the scope of this paper.) For long poly[2-methoxy, 5-(2'-ethylhexyloxy)-p-phenylene-vinylene] (MEH-PPV) polymers, it was found that the chain conformations are strongly dependent on the sample preparation as well as local environment. On the one hand, a poor solvent or a high molecular weight host matrix encourages packing of the chains. ${ }^{11,15,16}$ In this conformation, energy was found to funnel efficiently to a few lower transition energy exciton traps. On average only two to three discrete emitters exist in the collapsed-chain configuration. ${ }^{17}$ On the other hand, a good solvent or a low molecular weight host matrix leads to extended chain conformations. ${ }^{11,15}$ Many independent emitting sites were found within a extended conjugated polymer. ${ }^{17}$ In order to study exciton migration along the polymer backbone and rule out the complexities of solvent and local environment effects, Nguyen et al. incorporated MEH-PPV molecules into the nanopores of a silica composite showing that ET along the polymer chain is orders of magnitude slower than that between parallel-oriented chain-chain segments. ${ }^{12}$ Wang et al. found that the exciton migration mechanism and photophysics in persistent-length poly(2,5-dioctyloxy, $p$-phenylene-vinylene) (DOO-PPV), which has a rod-shape conformation, is substantially different from a long chain, folded polymer. By comparing the experimentally observed 
spectral fluctuation with that calculated from a molecular exciton model, they showed that migration of thermalized excitons is inefficient along the polymer backbone. To further eliminate thermal effects, low-temperature experiments have been performed on conjugated polymers. ${ }^{18-20}$ For longchain MEH-PPV, peaks in the fluorescence spectra were found to narrow and redshift by $\sim 20 \mathrm{~nm}$ relative to room temperature at $20 \mathrm{~K}$, indicating an increased effective conjugation length, ${ }^{18}$ corresponding to the exciton being delocalized over more repeat units of the polymer chain. This was attributed to the freezing out of the torsion modes of polymer chains at low temperature. Furthermore, spectral diffusion was found to be substantially stronger than at room temperature with an average range of $500 \mathrm{~cm}^{-1}$ and a maximum registered value up to $1100 \mathrm{~cm}^{-1}$ in the "host matrix free" environment. ${ }^{19}$

Since most experiments were performed with long chain polymers, the results reflect the statistical behavior of hundreds or thousands of absorbing chromophores. While the work in short DOO-PPV polymers checked ET along the backbone, the number of excitons in a polymer is still too large to unambiguously study the exciton energy migration between chromophores in a single polymer. In this paper we will detail work in which we extracted short-chain MEHPPV polymers, which have a rodlike conformation and contain only two to three chromophores, from pristine $\mathrm{MEH}-$ PPV powder. In such simple systems, the three dimensional intrachain ET can be ruled out and the one dimensional intrachain interaction of chromophores monitored. ET between chromophores within such systems was found to resemble a donor-acceptor system in which energy transfers from donors to an acceptor until the acceptor dies, after which, a donor takes turns to emit.

\section{EXPERIMENTS}

\section{A. Extraction of low molecular weight polymers}

Low molecular weight polymers were obtained from commercial MEH-PPV (SIGMA-ALDRICH, 541443) by means of a reprecipitated method. Pristine MEH-PPV powder was dissolved in toluene $(1 \mathrm{~g}: 100 \mathrm{ml})$ and stirred at $50{ }^{\circ} \mathrm{C}$. After two days, methanol was slowly added into the solution until precipitate appeared. Centrifugal sedimentation was used to remove the precipitate. This was repeated twice, after which the addition of methanol prompted no further precipitation. The solution (MEH-PPV in methanol and toluene) was dried by rotary decanter. The residual MEH-PPV was redissolved in toluene. Methanol was again added to the solution and the resulting gelatinoids were separated from the solution by centrifugal sedimentation. After washing with low temperature methanol and vacuum filtering, the final product was put into a vacuum oven for complete drying. The molecular weight $\left(M_{w}\right)$ of the final product as measured by gel permeation chromatography (GPC) was $\sim 7.8 \mathrm{kDa}$ with polydispersity $(\delta) \sim 1.8$.

\section{B. Ensemble measurements}

Ensemble measurements were made using dilute solutions of the polymer dissolved in chloroform. Absorption and PL spectra were measured using commercial units (GBC, Cintra-20 and Jobin Yvon, FL3-21 respectively). Fluorescence decay curves were recorded using a mode-locked tunable Ti:sapphire laser (Coherent, Mira 900 operating at $\lambda$ $=900 \mathrm{~nm}, 76 \mathrm{MHz}$ pulse rate) passed through a second harmonic generator to generate the $\lambda=450 \mathrm{~nm}$ as the excitation light source and measured with a streak camera (Hamamatsu, C5680-01).

\section{Single molecule spectroscopic studies}

For single molecule studies, the low molecular weight MEH-PPV powder was dissolved in chloroform solution and subsequently diluted with another chloroform solution containing polystyrene and spin cast onto a fused silica substrate. The film was protected from oxygen by sealing in a nitrogen glove box. The dilution factor was selected so that single polymers were well separated and could clearly be resolved. Observation was made using a homemade samplescanning confocal microscope $\mathrm{e}^{13}$ in epifluorescence mode. For CW measurements, an air-cooled Argon laser operating at $\lambda=488 \mathrm{~nm}$ was used as the excitation source while for pulse excitation the output of the frequency doubled Ti:sapphire laser $(\lambda=450 \mathrm{~nm})$ was used. The output of both lasers was linearly polarized and was focused to a $\sim 0.16 \mu \mathrm{m}^{2}$ spot by a fluorescence objective (Nikon, 100×, 1.3 NA). The average power of the irradiance on the sample plane was chosen to be $\sim 200 \mathrm{~W} / \mathrm{cm}^{2}$, on the lower end of the 200 $1600 \mathrm{~W} / \mathrm{cm}^{2}$ commonly used for single polymer experiments, ${ }^{13,21}$ is well below the exciton saturation intensity $\left(\sim \mathrm{MW} / \mathrm{cm}^{2}\right)$, ensuring that the fluorescence lifetime is not affected by the intensity. PL was collected by the same objective and detected by an avalanche photodiode (APD) operating in single photon counting mode (PerkinElmer, SPCM-AQR-15-FC). After determining the positions of single polymers by raster scanning the sample under low irradiance, the excitation beam was fixed on individual polymers and the PL intensity fluctuation recorded with $10 \mathrm{~ms}$ time resolution.

The conformation of the short chain polymers were studied by slowly rotating the polarization of the $\mathrm{CW}$ excitation light. ${ }^{22}$ Excitation light was passed, in order, through a polarizer, an electrooptical modulator, and a quarter-wave plate. The polarization direction was modulated between 0 and $\pi$ with a period of $0.2 \mathrm{~s}$ and the PL intensity recorded as a function of polarization angle and time. For rodlike polymers lying in the sample plane, all chromophores on the polymer have the same orientation. They will not be excited when the polarization of the excitation light is perpendicular to them. Therefore, a $100 \%$ modulation of the PL intensity is expected. ${ }^{21}$

In order to monitor the time-dependent spectral change, a polarization insensitive beam splitter $\left(\lambda_{\text {center }}=555 \mathrm{~nm}\right)$ was used to split the fluorescence between two APDs. The variation of the PL spectrum over time was then directly monitored by calculating the normalized spectral shift coefficient, $S$, which is defined as ${ }^{13}$ 


$$
S=\frac{\gamma I_{\text {red }}-I_{\text {blue }}}{\gamma I_{\text {red }}+I_{\text {blue }}}
$$

Here $I_{\text {red }}$ and $I_{\text {blue }}$ are intensities of red and blue ports, respectively, and $\gamma$ is the ratio of the detection efficiencies of the two ports ( $1 / 2$ for our system).

To measure the correlation of photons emitting from a single polymer, the temporal separation between consecutive photons of the two APDs was measured by the HanburyBrown and Twiss photon correlation experimental scheme ${ }^{23}$ using the pulsed laser source. ${ }^{24}$ In our implementation, a Soleil-Babinet compensator (New Focus, 5540) operating as a quarter-wave plate was used to transform the linearpolarized output of the pulsed laser into circular-polarized light to allow chromophores in polymers with different orientations can be excited equally. The PL from the sample was split by a $50 / 50$ beam splitter and directed into two separate APD detectors. A time-correlated single-photon counting (TCSPC) module (Becker \& Hickl GmbH, SPC600 ) was used to measures the time intervals between adjacent photons. The time intervals were ordered in a histogram to get the interphoton time distribution from which the number of emitters can be obtained. ${ }^{24,25}$ For a single emitter, the probability of emitting two consecutive photons drops to zero for time intervals shorter than the excited-state lifetime as a chromophore cannot emit two photons simultaneously (photon antibunching). For two, three, and four independent emitters, the probabilities are $0.5,0.67$, and 0.75 , respectively. Using pulse excitation, interphoton times will distribute around the multiples of the reciprocal of the repetition rate of the excitation pulsed laser with the peak at zero interphoton time presenting the number of photon pairs that excited by the same laser pulses and the other peaks being the photon pairs excited by different laser pulses.

Fluorescence decay curves were obtained using the same pulsed laser and TCSPC module. Part of the laser pulse was directed to a silicon photodiode which acted as a reference signal while the remainder excited the sample. The emitted light was detected using a photon counting GaAsP PMT (Hamamatsu, H7422P-40, time resolution $=300 \mathrm{ps}$ ) as it has better temporal stability than APDs. The fluorescence time trace was reconstructed by binning the arrival times of the photons. The time intervals between the arrival of emitted photons and the reference signal were used to construct fluorescence decay curves as a function of time.

\section{RESULTS AND DISCUSSION}

\section{A. Sample characterization}

The absorption and PL spectra for short-chain MEH-PPV (black curves) compared to regular long-chain MEH-PPV $\left(M_{w}=210 \mathrm{kDa}\right.$, gray curves) in chloroform are shown in Fig. 1. Both the absorption and PL spectra of short-chain MEHPPV are blueshifted relative to the long-chain polymer. The peak of the absorption spectrum has shifted much more than the peak of the PL spectrum (15 nm vs $4 \mathrm{~nm}$, respectively). The difference in absorption spectra indicates that the conjugation length distribution of the short-chain polymers is more biased toward the short segments than that of the long-

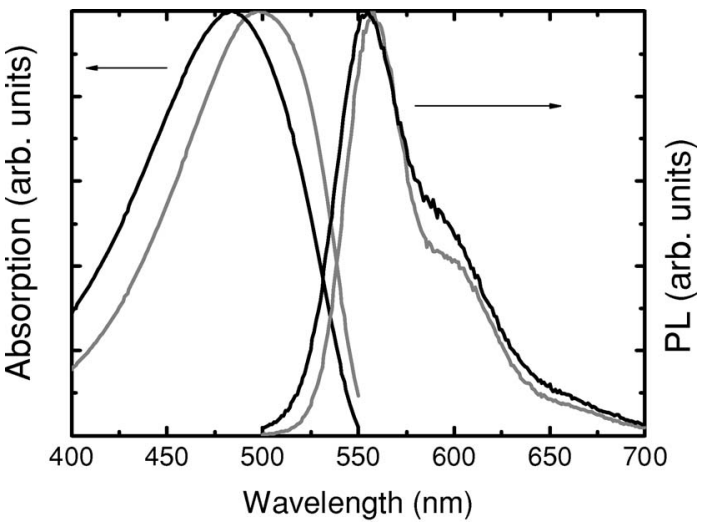

FIG. 1. Ensemble absorption and PL intensity spectra of shortchain (black) and long-chain (gray) MEH-PPV polymers in chloroform.

chain polymers. Because excitons migrate to lower energy sites before emitting light, the PL spectra is only sensitive to the longest conjugated segments. As a result the wavelength difference between the peaks of PL spectra for short- and long-chain polymers is smaller than that of absorption spectra.

\section{B. Single molecule fluorescence time trace}

Typically, in MEH-PPV, each chromophore (conjugated segment) is composed of about 10-15 adjacent repeat units. ${ }^{26}$ Taking into account the polydispersity $(\delta)$ of our polymer and the conjugation length distribution, we estimate the number of chromophores in a single molecule of our samples to be between one and three. For an individual polymer, the number of absorption chromophores in a polymer should be reflected by steps in its fluorescence time trace. A typical trace for the short chain polymers is shown in Fig. 2. Changes between intensity levels are abrupt (within the $10 \mathrm{~ms}$ time resolution of our system). Three discrete intensity levels can be recognized in this time trace indicating the presence of three active absorbing chromophores (indicated by dashed lines in Fig. 2). This multiple discrete-level emission is the main feature of a multichromophoric system. For

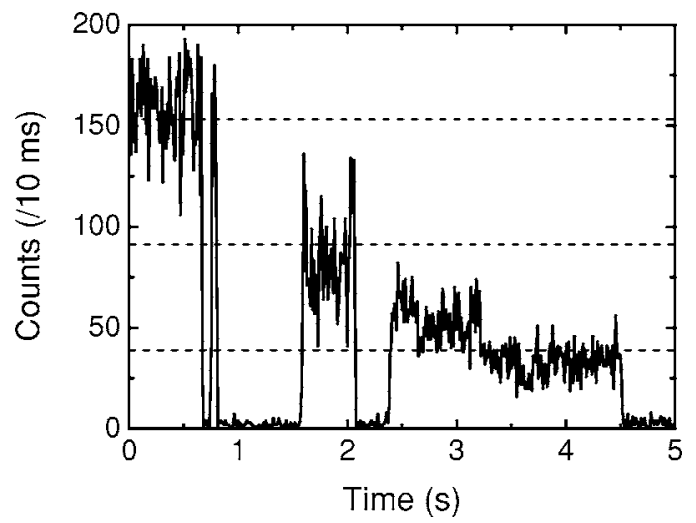

FIG. 2. Typical fluorescence time trace for a single short-chain polymer. Data was taken under constant excitation irradiance $\left(\sim 200 \mathrm{~W} / \mathrm{cm}^{2}\right)$. 
the 163 single MEH-PPV polymers studied, $40 \%, 42 \%$, and $18 \%$ of time traces of single molecules were found to exhibit three, two, and one discrete levels, respectively. A significant feature of these short-chain curves is the fact that the intensity always drops to or very close to the background level before rebounding to emit at a different intensity. This is in stark contrast to short DOO-PPV polymers, ${ }^{13}$ in which the fluorescence time traces do not directly drop to the background level. This "on/off" blinking behavior observed in short-chain MEH-PPV is a significant feature of single emitter, just like in a single dye molecule. Since the multiplelevel behavior infers that there are about 2-3 chromophores in a MEH-PPV polymer, the one-emitter feature implies that the energies of excitons are transferred to the lowest transition energy site before they are emitted. While ET along the backbone has been shown experimentally to be inefficient in comparison to that between three dimensional chain-chain segments, theoretical calculations ${ }^{14,27}$ based on the Förster theory indicate that the intrachain ET rate between short and longer chromophores along the backbone, while slower than three dimensional ET, is still of the order of $0.01 \mathrm{ps}^{-1}$. This value is at least an order of magnitude larger than the fluorescence decay rate of short-chain MEH-PPV, which is the order of $0.001 \mathrm{ps}^{-1}$. For long-chain MEH-PPV molecules in silica nanopores ${ }^{12}$ and persistent-length DOO-PPV polymers, energies of excitons are transferred along polymer backbone to local minimum sites in their vicinity rather than to the global minimum site before emission.

In addition to the observed blinking behavior, the number of simultaneous emitters can further be checked via their interphoton time statistics. ${ }^{24}$ On the one hand, if the two or three chromophores emit independently, there should be multiple emitting sites on a single molecule. On the other hand, if all the chromophores transfer their energy efficiently to the lowest transition energy site, there should be only one emitting site at one time. Figure 3(a) shows the distribution of interphoton times for a single MEH-PPV polymer. Interphoton times distribute around the multiples of $13 \mathrm{~ns}$ which corresponds to the repetition rate of the pulsed laser. The value of the zero-interphoton-time peak is very small compared to those of other peaks. The probability of emitting two photons simultaneously equals to the ratio of the zero-interphotontime peak to the average of the other peaks. From the figure, this ratio is almost zero, which indicates that any given time only one emitter is active in this polymer. Similar results were seen for the majority of other polymers. Figure $3(\mathrm{~b})$ shows the distribution of interphoton times summed over 69 single molecules. As seen in the figure, the height of the zero-interphoton-time peak is very small relative to the other peaks, confirming that in the majority of short chain polymers, only one chromophore emits at a given point in time. This implies that there is both efficient energy transfer as well as exciton-exciton annihilation in this system. ${ }^{25}$ (The latter is not unexpected as exciton-exciton annihilation has been previously reported to be efficient in PPV derivatives. ${ }^{28,29}$ ) In summary, the above experimental results are consistent with that of a short rodlike MEH-PPV having one or more absorbing chromophores transferring energy to a single emitting chromophore at any given point in time.
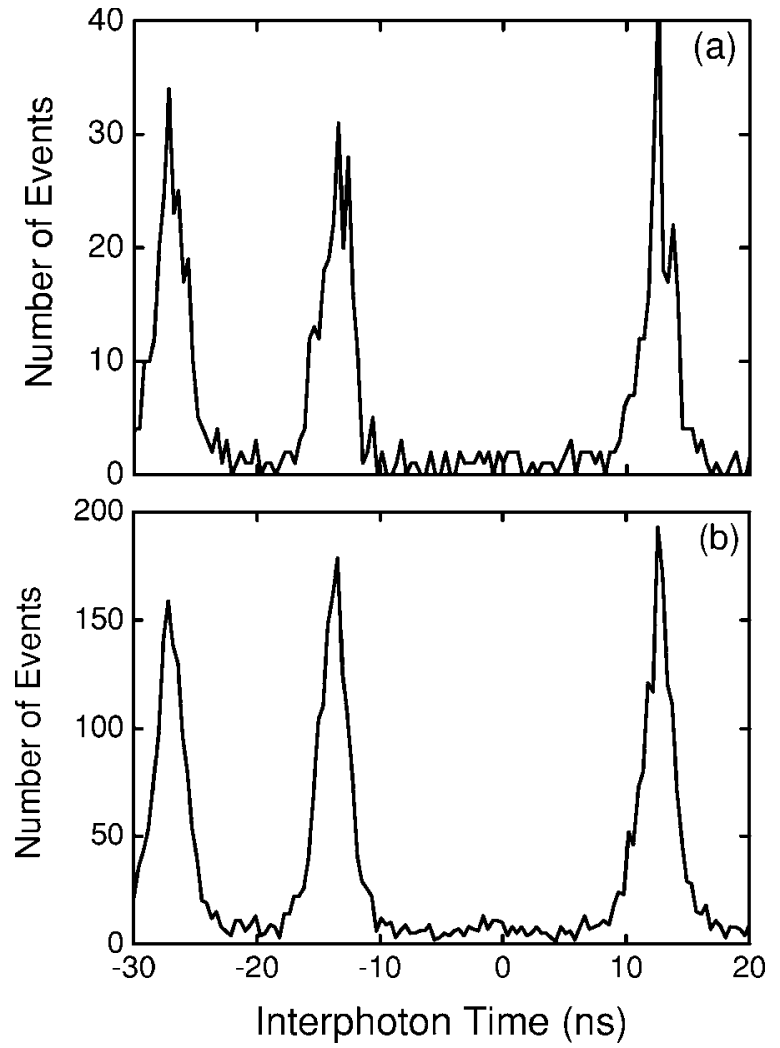

FIG. 3. Interphoton time histogram for (a) a single short-chain polymer and (b) the coherent sum (not ensemble measurement) of 69 single polymers.

\section{Confirmation of rodlike polymer conformation}

As the short-chain MEH-PPV polymers investigated here are close to the persistence length, ${ }^{30}$ they are expected, in the absence of defects, to take a predominately rodlike conformation with the dipoles of the absorption chromophores aligned. The conformation was verified experimentally by means of polarization spectroscopy. ${ }^{22}$ Figure 4 shows a typical result for a single polymer. As seen in the figure, the fluorescence intensity is modulated at the same frequency as the polarization of excitation light. The fully modulated na-

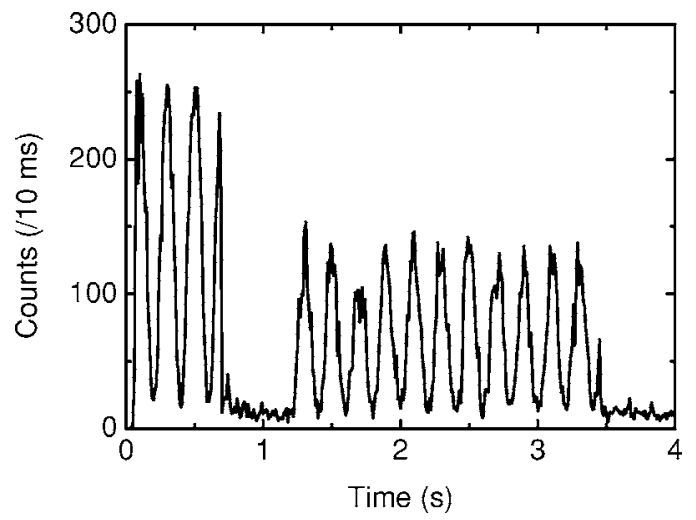

FIG. 4. Typical fluorescence time trace for a single short-chain polymer when the polarization of the excitation light is modulated at $5 \mathrm{~Hz}$. 


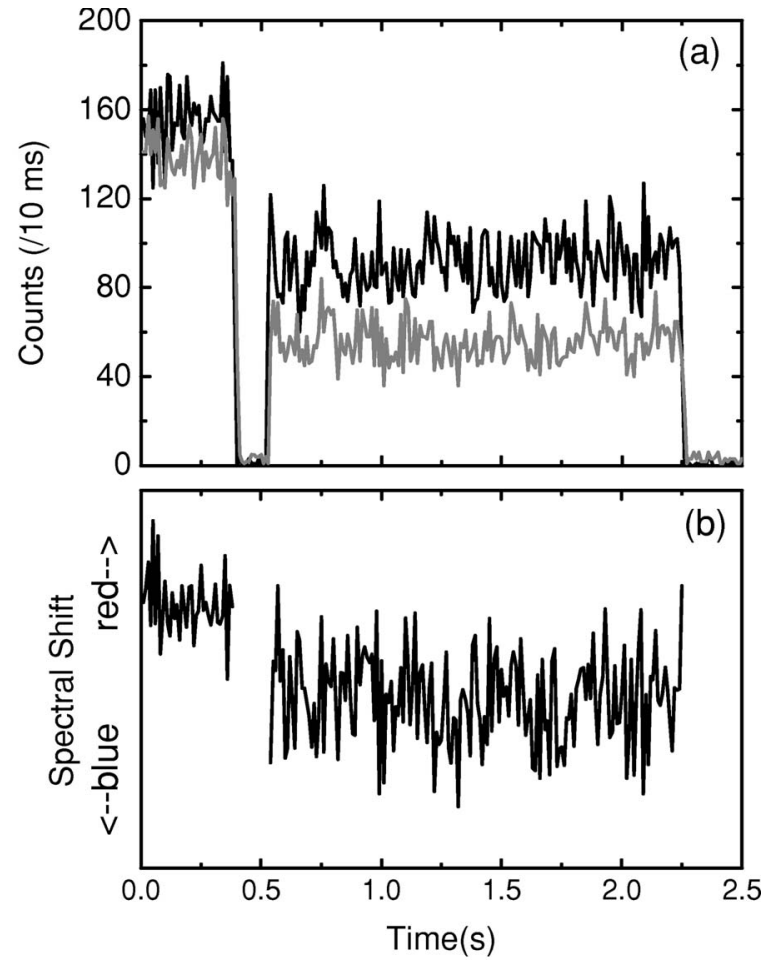

FIG. 5. (a) Fluorescence time trace for a single short-chain polymer where the fluorescence was split at $\lambda=550 \mathrm{~nm}$ and directed onto two APDs. The count rates recorded by the APDs monitoring the red $(\lambda>550 \mathrm{~nm})$ and blue $(\lambda<550 \mathrm{~nm})$ portions of the spectrum are indicated by gray and black lines respectively. (b) Spectral shift coefficient (see text for details) indicating changes in the spectral composition of the fluorescence.

ture of the time trace (with the fluorescence dropping to the background level every half-cycle) indicates that the shortchain polymer does indeed have a rodlike conformation and that all the absorption dipoles aligned. As a result, exciton energy transfer, if it occurs, is restricted to be solely along the polymer backbone.

\section{Characterization of the emission site trajectory}

Changes to the properties of the emission chromophore in a single polymer were tracked by monitoring changes in the spectral composition and fluorescence lifetime during its survival time. The crucial question to be addressed here is whether, within a single polymer, the same emission site is responsible for emission both before and after a blinking event (i.e., For the single polymer in Fig. 2, is the chromophore emitting from the same one as was emitting before the blinking event or is it a different chromophore?) If it is the same chromophore, then one expects the emission spectrum to be the same both before and after the blinking event. Any change in emission site will be accompanied by a shift in PL spectrum. If the emitting site transfers to a site with higher (lower) transition energy after a blinking event, the PL spectrum will blueshift (redshift). Figure 5 illustrates a typical fluorescence time trace and spectral shift for a single polymer. From Fig. 5(a) it is clear that the same blinking behavior is seen in both two channels with short term (i.e.,
10-100 ms) fluctuations roughly correlated. There is, however, a significant difference between the two intensity levels before and after the blinking event. This is seen more clearly in Fig. 5(b), where the spectral shift coefficient is seen to be quite different before and after the blinking event indicating that the emitting chromophores at earlier and latter times are different. In this case the emitting chromophore at the second step has larger transition energy than that at the first step. For the 98 single polymers studied, the fluorescence of $60 \%$ of polymers clearly shifted to the blue after a blinking event, $21 \%$ exhibited spectral shifts less than the signal-to-noise ratio of our detection system, and in the remaining 19\% the spectrum shifted to the red. This suggests that while for the majority of polymers the emission site changes after a blinking event, up to $21 \%$ of the time either the same chromophore resumes emission after recovery from a temporary quench event or the energy difference between emitting sites before and after blinking event is too small to be resolved with the spectral shift method.

Changes in the emitting chromophore are also reflected by changes in the fluorescence lifetime. If it is the same chromophore, then one expects not only the emission spectrum as well as the fluorescence lifetime to be the same both before and after the blinking event. Any change in emission site will be accompanied by not only a shift in PL spectrum but also a change in fluorescence lifetime. Relative to directly observing the emission spectrum, this offers the advantage of independence of PL intensity and higher signal to noise ratio.

According to exciton theory, ${ }^{7}$ a conjugated system of linear geometry and $N$ units the energy of the exciton state with largest transition moment is given by

$$
E=E_{0}+2 \beta \cos \left(\frac{\pi}{N+1}\right),
$$

where $E_{0}$ is the excited state of each unit and $\beta$ is the interaction strength between nearest-neighbor units and is a negative value. According to this equation, the energy of the exciton state decreases with increased chain length. While the energies of emitting excitons can be checked by monitoring the fluorescence spectra, they can also be checked by observing the fluorescence lifetime $\left(\tau_{f}\right)$. The latter is related to both the radiative $\left(k_{r}\right)$ and nonradiative $\left(k_{n r}\right)$ rate constants by

$$
\tau_{f}=\frac{1}{k_{r}+k_{n r}} .
$$

$k_{r}$ is in turn equal to the Einstein spontaneous emission coefficient $(A)$, where ${ }^{31}$

$$
k_{r}=A \propto\left|\mu_{N}\right|^{2} .
$$

Here $\left|\mu_{N}\right|$ is the transition dipole moment for a conjugated segment of $N$ units and can be expressed as

$$
\left|\mu_{N}\right|^{2}=\frac{2|\mu|^{2}}{N+1}\left[\cot \left(\frac{\pi}{2 N+2}\right)\right]^{2},
$$

where $|\mu|$ presents the dipole moment for each unit. This equation predicts that $\left|\mu_{N}\right|$ increases with increasing conjugation length. Although $\tau_{f}$ measured by the time-resolved PL includes contributions from both $k_{r}$ and $k_{n r}$, the increase of 


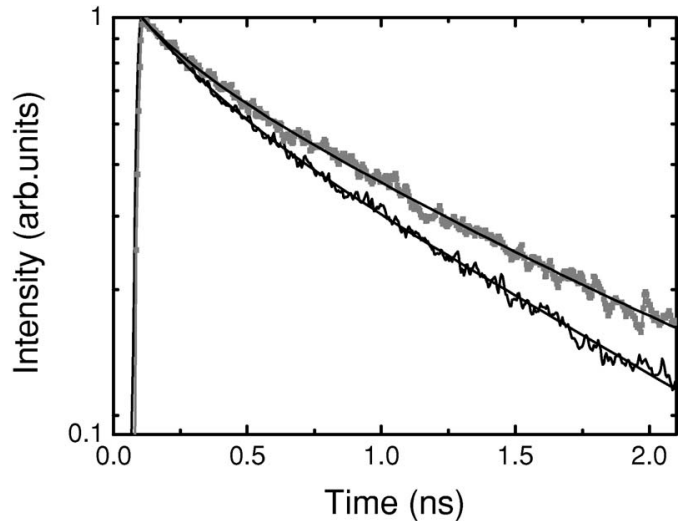

FIG. 6. Ensemble fluorescence decay curves of short-chain MEH-PPV polymers in chloroform measured before extended excitation (black) and after the sample was continuously excited at $\lambda$ $=488 \mathrm{~nm}$ for $60 \mathrm{~min}$ (gray). The smooth curves represent the results of fitting with a two-exponential decay function.

transition dipole moment with conjugation length is reflected by a reduction in $\tau_{f}$ as is apparent from Eqs. (3)-(5). Indeed, just such a progressive decrease in $\tau_{f}$ was found in a series of oligomers with an increasing number of phenyl rings. ${ }^{32}$ Therefore, it is reasonable to attribute changes in the fluorescent lifetime to changes of conjugation length of emitting chromophores. As indicated by Eqs. (2) and (5), chromophores with larger transition energies have shorter effective conjugation lengths (smaller $N$ ) and smaller $\left|\mu_{N}\right|$. Hence, according to Eq. (4), a change in emission site from a lower (longer conjugation length) to higher (shorter conjugation length) exciton energy site will result in an abrupt increase in the fluorescent lifetime. Therefore, the blueshift (redshift) in the PL spectrum will be accompanied by an increase (decrease) in fluorescence lifetime.

Figure 6 shows the ensemble fluorescence decay curves of a solution of short MEH-PPV polymers after different excitation times. The black curve was obtained before extended excitation and the gray curve was measured after the sample was continuously excited for $60 \mathrm{~min}$. Both curves were fitted with a two-exponential decay function. The lifetimes for the black curve were $0.21 \mathrm{~ns}$ and $1.17 \mathrm{~ns}$ with amplitudes of 0.30 and 0.85 while the gray curve was characterized by lifetimes of $0.24 \mathrm{~ns}$ and $1.35 \mathrm{~ns}$ with amplitudes of 0.34 and 0.83 . The longer lifetimes at the later time is indicative of a shortening of the average conjugation length of the emitting segments. This is consistent with the picture that excited chromophores transfer their energy to the available lowest transition energy sites. Thus, during excitation, the lowest transition energy (shortest lifetime) chromophores are preferentially quenched, resulting in an increase in the average energy (and lifetime) as the sample photobleaches.

The argument can also be supported by comparing the ensemble absorption and PL spectra of a fresh sample with that after an extended period of continuous excitation (Fig. 7). Absorption at longer wavelengths decreases over time while at the same time there is a slight increase at higher energies $(\lambda<400 \mathrm{~nm})$. This is consistent with a preferential quenching of longer conjugation length chromophores. The increase in the blue portion suggests the creation of shorter

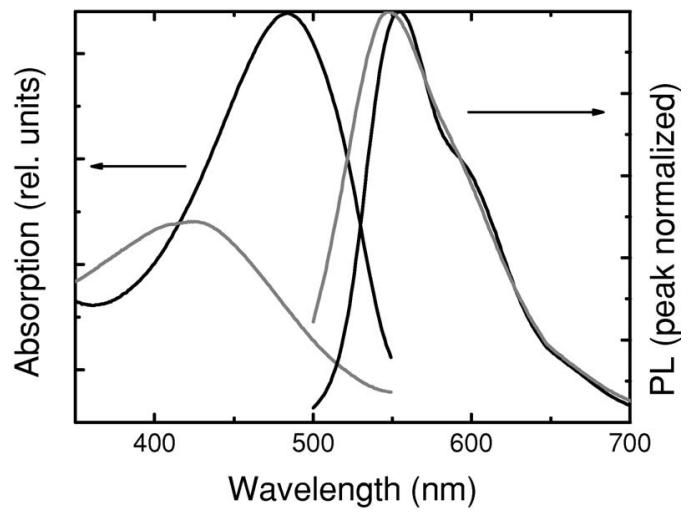

FIG. 7. Ensemble absorption and PL spectra for short-chain MEH-PPV polymers in chloroform measured before extended excitation (black) and after an extended period of continuous excitation (gray) at $\lambda=488 \mathrm{~nm}$. The absorption spectra are relative intensities while the PL spectra are peak normalized.

conjugated segments-most likely the result of $\mathrm{C}=\mathrm{O}$ bonds interrupting longer conjugated segments. The PL spectra (peak normalized in Fig. 7), which reflects the emission properties of the longest unquenched conjugated segment, also becomes slightly blue shifted over time. Taken together, the absorption and PL spectra confirm both a shortening of the average conjugation length of the absorbing chromophores along with a shortening of the conjugation length of the emitting chromophores.

It is interesting to compare the fluorescence lifetime values reported for these short-chain polymers with that of longchain polymers under similar conditions. Typically, the fluorescence lifetime for long-chain MEH-PPV polymer in solution is about $330 \mathrm{ps}$ (Ref. 33) - a factor of $\sim 3$ times shorter than that obtained here for short chain polymers. We attribute the longer lifetime of the shorter polymers to their simple rodlike conformation that excludes most nonradiative processes caused by complex chain conformations. We note that similar lifetime changes have been observed in chainlength dependence experiments for DOO-PPV dissolved in chloroform. ${ }^{34}$ A secondary, and much less significant effect, results from the positive correlation of radiative decay rate with conjugation length coupled with the shorter average conjugation length in the polymers investigated here relative to long-chain polymers (cf., Fig. 1).

Changes in the fluorescence lifetime during photobleaching can also be directly observed in single polymers. Figure 8(a) illustrates a fluorescence time trace compiled by binning the arrival times of the collected photons. The corresponding fluorescence decay curves for the different PL intensity levels before and after a blinking event are shown in Figs. 8(b) and $8(\mathrm{c})$ as indicated by the arrows. The fluorescence lifetime was obtained by fitting the fluorescence decay curves with a single-exponential decay function. The fluorescence lifetimes before and after the blinking event were thus determined to be $1.22 \mathrm{~ns}$ and $1.38 \mathrm{~ns}$ respectively. The fluorescence lifetime for the second step is significantly larger than that for the first step suggesting that the emitter stays at a lower transition energy site until the blinking event and then transfers to a higher transition energy site after the original site is quenched. 


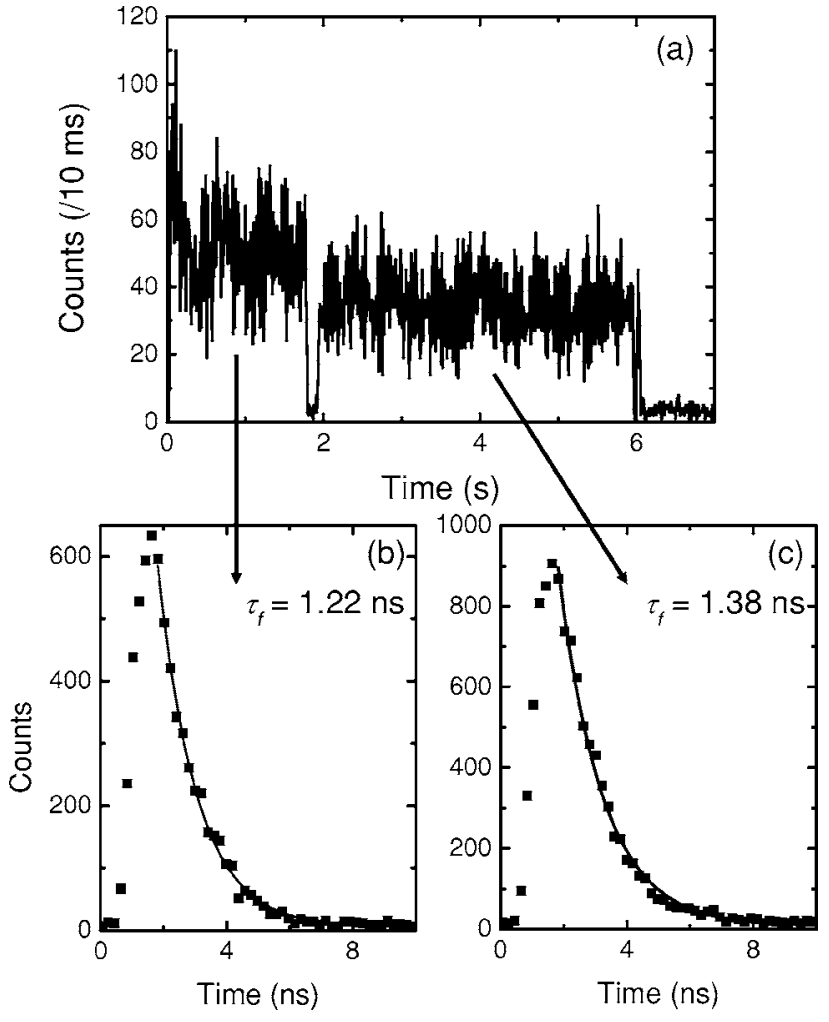

FIG. 8. Correlation between fluorescence intensity and fluorescence lifetime for a single short-chain polymer. (a) Fluorescence time trace formed by binning the arrival times of fluorescent photons into $10 \mathrm{~ms}$ bins. (b) Fluorescence decay curve at earlier time (high intensity). (c) Fluorescence decay curve at later time (low intensity). The period at which the fluorescence decay curves are taken is indicated by the arrows. In (b) and (c) the data points are illustrated by symbols with the solid lines denoting single exponential decay fitting curves. The corresponding lifetimes are $1.22 \mathrm{~ns}$ and $1.38 \mathrm{~ns}$, respectively.

The ratio of the fluorescence lifetimes after and before a blinking event $\left(\tau_{\text {after }} / \tau_{\text {before }}\right)$ can be used to characterize the migration of the emitting sites, (i.e., $\tau_{\text {after }} / \tau_{\text {before }}>1$ indicates a migration to a higher transition energy site, while $\tau_{\text {after }} / \tau_{\text {before }}<1$ indicates the emission changes to a lower transition energy site). A histogram of the fluorescence lifetime ratios for 57 spectral changes occurring in 49 molecules is shown in Fig. 9. The majority of values are greater than one (i.e., $\tau_{\text {after }}>\tau_{\text {before }}$ ) indicating an increase in fluorescent lifetime after the blinking event corresponding to a shift in emission from a lower to a higher transition energy chromophore. This is consistent with the spectra shift results indicating a shift in emission from longer conjugation length chromophores to shorter conjugated segments. In only three cases $(\sim 5 \%$ of the total) is there a clear decrease in the fluorescence lifetime. It is quite possible that, due to the polydispersity of the sample, these exceptional polymers have longer chain lengths supporting four or more absorbing chromophores and two independent emission sites. In a number of cases the ratio is $\sim 1$, this may be a result of either a temporary quench event or the case of two emitting chromophores before and after the blinking event having conjugated segments of approximately equal length.

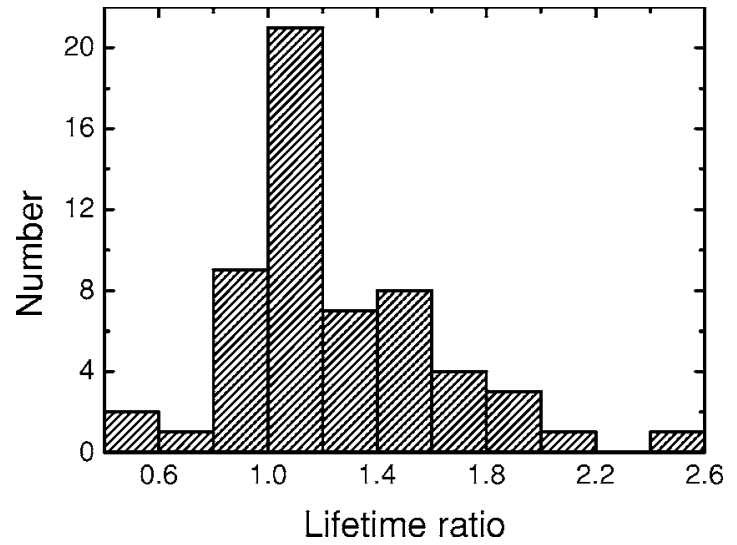

FIG. 9. Histogram of the fluorescence lifetime ratios after and before blinking events $\left(\tau_{\text {after }} / \tau_{\text {before }}\right)$ observed for 57 blinking events in 49 single polymers.

\section{E. Interpretation of the results}

To summarize the experimental results, in a single shortchain rodlike polymer of MEH-PPV, as the polymer photobleaches, the PL intensity drops in abrupt steps, separated by temporary drops of intensity to or close to the background level. With a change in the intensity of emitted light, there is usually a corresponding change in both the spectral composition and fluorescence lifetime. In general, emission at lower intensities occurs later in the polymer's survival time, and is both blueshifted and characterized by a longer fluorescence lifetime.

One way of interpreting these results is within the framework of a molecular exciton model. The on/off blinking behavior and the observed photon antibunching indicate that while there may be between one and three absorbing chromophores in the polymer, there is only one emitter at a given point in time. This suggests that exciton energy transfers from higher transition energy chromophores or shorter conjugated segments (donors) to a single lower transition energy chromophore or long conjugated segment (acceptor) along the polymer backbone (rodlike polymer) by Förster energy transfer before recombination and fluorescent emission. In classical donor-acceptor systems, fluorescence switching from acceptor to donor ${ }^{35}$ is observed after the former is quenched or photobleached. Similarly, in the majority of polymers observed here, the intensity, spectral and fluorescent lifetime measurements indicate that after a blinking event, emission either continues from the same site (temporary quench event) or resumes from a higher transition energy site (permanent quench event). In either case, the polymer spends the order of $\sim 100 \mathrm{~ms}$ in the dark state before emission resumes, suggesting that, in the case of a change in emission site, the chemical reaction leading to the quench event (i.e., formation of a $\mathrm{C}=\mathrm{O}$ group) is followed by some type of slower geometry change. This change acts to stop the transfer of energy to the lower transition energy chromophore, allowing a higher transition energy chromophore to start emitting. To summarize, energy transfer is always efficient enough that fluorescence is from the global energy minimum. The observed chromophore is always the longest 
chromophore in the polymer and it is impossible to know, from the PL spectra, the energies for the other chromophores until the longer ones are quenched and they become the only emitter.

Considerable work has been done studying the energy transfer within MEH-PPV and the closely related DOO-PPV. It is generally accepted that MEH-PPV forms a defect coiled structure $^{10}$ in solution and when deposited into a condensed phase matrix. Processing conditions have been found to strongly influence the "tightness" of the structure. ${ }^{15}$ While there are numerous absorbing segments or chromophores in a polymer, the number of emitting chromophores depends on the conformation. In tight configurations only a few or even a single emitting chromophore exists while in more extended configurations there can be hundreds of emitting chromophores. ${ }^{17}$ Correlated quantum-chemical calculations for polyindenofluorene indicate that energy transfer between closely packed chains can be orders of magnitude higher than energy migration along the polymer backbone. ${ }^{27} \mathrm{Im}$ planting single chains of MEH-PPV into the channels of oriented, hexagonal nanoporous silica has given experimental support for these calculations. ${ }^{12}$ The crucial factor determining energy transfer efficiency and hence the number of emission sites is the Förster radius. When chromophore separation is less than the Förster radius, energy transfer is efficient resulting in fewer emission sites. On the basis of the Förster radius, tightness can be placed on a more quantitative basis. When interchain separation is less than the Förster radius, three-dimensional energy transfer dominates resulting in few deep or low energy emission sites. When interchain separation is greater than the Förster radius, the slower intrachain energy transfer dominates and there are many shallow emission sites of approximately equal energy as the excited state lifetime is not sufficiently long to allow excitons to move to the globally lowest energy emission site. ${ }^{36}$ Work on rodlike DOO-PPV found multiple emission sites implying that for as few as four or five conjugated segments, excitons do not have always have sufficient time to migrate to the globally lowest energy emission site before emission but rather settle into local minimum. ${ }^{13}$ The present work indicates that for polymers with less than four conjugated segments, excitons have sufficient time to migrate to the lowest available emission site. The Förster radius is crucially dependent on spectral overlap between adjacent conjugated segments. Calculations based on absorption and PL spectrums obtained from a molecular exciton model, ${ }^{7}$ suggest that while strong spectral overlap exists between the emission of short conjugated segments and the absorption of long conjugated segments, the reverse is not necessarily true. The implication is that while an exciton can rapidly migrate to lower energy sites, short conjugated segments (representing an uphill transition) significantly slow down intrachain migration. The present work has provided further insight into the distance an exciton can travel along a single chain during its lifetime. Along with the rodlike DOO-PPV results the present work provides both upper and lower bounds for the Förster radius for intrachain energy transfer.
For both short rodlike polymers and tightly coiled sections of a long polymer only one emitter exists. The result of a permanent quench event is, however, quite different. In the first case, after a short dark period, emission simply switches to another segment, while in the latter, emission from the whole section is quenched.9,17,37 One possible explanation for the different behavior is that in the case of a tightly coiled polymer multiple pathways exist for energy transfer while for a rodlike structure there is only one pathway. Thus in the case of rodlike structure, a single geometrical or conformational change (associated with quenching) could effectively block the pathway. Along with spectral overlap, conformation (i.e., relative angles of the dipoles) also affects the Förster radius. ${ }^{38}$ For example, when a polymer is completely free-as in solution - an oxygen quench event results in the formation of a tetrahedral defect $\left(120^{\circ}\right.$ change in bond angle) thus reducing the Förster radius significantly. Under partially constrained conditions (i.e., short polymer), it is not unreasonable also to expect some conformation change. The length of time the molecule remains in the dark state, $\sim 100 \mathrm{~ms}$, suggests a time frame for this reorganization. Clearly in the case of a long-chain polymer, especially one that is tightly coiled, any such geometrical reorganization would require a considerably longer time frame, although recent studies seem to indicate that this occurs, at least for some polymers. ${ }^{39,40}$

In contrast, the optical signature of temporary quench events (i.e., emission recovers to its original intensity level and spectral composition) within short chain MEH-PPV is similar to that in tightly coiled sections of long polymers. Insight into temporary quench events, given by modulating the electric field, ${ }^{41,42}$ suggest that such a temporary quench may be the result of the charges inducing exciton disassociating. In this case there is no permanent chemical or conformational change.

\section{CONCLUSION}

Since the dynamics of conjugated polymers is extremely complex in comparison to that of a simple organic dye, in this paper, short-chain rodlike MEH-PPV polymers, containing from one to three chromophores or conjugated segments, were used to study energy transfer between chromophores and monitor changes in the emission with time. The key conclusion of this work is that the two or three chromophores in a single rodlike conjugated polymer interact like a classical donor-acceptor system in which energy is transferred to the acceptor until it bleaches, after which a donor starts to emit.

\section{ACKNOWLEDGMENTS}

We thank J. J. Liang for useful discussions. This research was partially supported by the National Science Council, Taiwan, under project number NSC 94-2112-M-001-038. 
${ }^{1}$ A. J. Heeger, Angew. Chem., Int. Ed. 40, 2591 (2001).

${ }^{2}$ G. Malliaras and R. Friend, Phys. Today 58, 53 (2005).

${ }^{3}$ N. S. Sariciftci, Primary Photoexcitations in Conjugated Polymers: Molecular Exciton Versus Semiconductor Band Model, 1st edition (World Scientific, New York, 1997).

${ }^{4}$ P. F. Barbara, A. J. Gesquiere, S. J. Park, and Y. J. Lee, Acc. Chem. Res. 38, 602 (2005).

${ }^{5}$ G. D. Scholes and G. Rumbles, Nat. Mater. 5, 920 (2006).

${ }^{6}$ L. J. Rothberg, M. Yan, F. Papadimitrakopoulos, M. E. Galvin, E. W. Kwock, and T. M. Miller, Synth. Met. 80, 41 (1996).

${ }^{7}$ R. Chang, J. H. Hsu, W. S. Fann, K. K. Liang, C. H. Chiang, M. Hayashi, J. Yu, S. H. Lin, E. C. Chang, K. R. Chuang, and S. A. Chen, Chem. Phys. Lett. 317, 142 (2000).

${ }^{8}$ W. T. Yip, D. H. Hu, J. Yu, D. A. Vanden Bout, and P. F. Barbara, J. Phys. Chem. A 102, 7564 (1998).

${ }^{9}$ J. Yu, D. H. Hu, and P. F. Barbara, Science 289, 1327 (2000).

${ }^{10}$ D. H. Hu, J. Yu, K. Wong, B. Bagchi, P. J. Rossky, and P. F. Barbara, Nature (London) 405, 1030 (2000).

${ }^{11}$ T. Huser, M. Yan, and L. J. Rothberg, Proc. Natl. Acad. Sci. U.S.A. 97, 11187 (2000).

${ }^{12}$ T. Q. Nguyen, J. J. Wu, V. Doan, B. J. Schwartz, and S. H. Tolbert, Science 288, 652 (2000).

${ }^{13}$ C. F. Wang, J. D. White, T. L. Lim, J. H. Hsu, S. C. Yang, W. S. Fann, K. Y. Peng, and S. A. Chen, Phys. Rev. B 67, 035202 (2003).

${ }^{14}$ D. Beljonne, G. Pourtois, C. Silva, E. Hennebicq, L. M. Herz, R. H. Friend, G. D. Scholes, S. Setayesh, K. Mullen, and J. L. Bredas, Proc. Natl. Acad. Sci. U.S.A. 99, 10982 (2002).

${ }^{15}$ S. S. Sartori, S. De Feyter, J. Hofkens, M. Van der Auweraer, F. De Schryver, K. Brunner, and J. W. Hofstraat, Macromolecules 36, 500 (2003).

${ }^{16}$ T. Huser and M. Yan, J. Photochem. Photobiol., A 144, 43 (2001).

${ }^{17}$ C. W. Hollars, S. M. Lane, and T. Huser, Chem. Phys. Lett. 370, 393 (2003).

${ }^{18}$ Z. H. Yu and P. F. Barbara, J. Phys. Chem. B 108, 11321 (2004).

${ }^{19}$ T. Pullerits, O. Mirzov, and I. G. Scheblykin, J. Phys. Chem. B 109, 19099 (2005).

${ }^{20}$ J. G. Muller, U. Lemmer, G. Raschke, M. Anni, U. Scherf, J. M. Lupton, and J. Feldmann, Phys. Rev. Lett. 91, 267403 (2003).

${ }^{21}$ J. D. White, J. H. Hsu, W. S. Fann, S. C. Yang, G. Y. Pern, and S. A. Chen, Chem. Phys. Lett. 338, 263 (2001).

${ }^{22}$ T. Ha, J. Glass, T. Enderle, D. S. Chemla, and S. Weiss, Phys. Rev. Lett. 80, 2093 (1998).
${ }^{23}$ R. H. Brown and R. Q. Twiss, Nature (London) 177, 27 (1956).

${ }^{24}$ K. D. Weston, M. Dyck, P. Tinnefeld, C. Muller, D. P. Herten, and M. Sauer, Anal. Chem. 74, 5342 (2002).

${ }^{25}$ P. Tinnefeld, K. D. Weston, T. Vosch, M. Cotlet, T. Weil, J. Hofkens, K. Mullen, F. C. De Schryver, and M. Sauer, J. Am. Chem. Soc. 124, 14310 (2002).

${ }^{26}$ H. Meier, U. Stalmach, and H. Kolshorn, Acta Polym. 48, 379 (1997).

${ }^{27}$ E. Hennebicq, G. Pourtois, G. D. Scholes, L. M. Herz, D. M. Russell, C. Silva, S. Setayesh, A. C. Grimsdale, K. Mullen, J. L. Bredas, and D. Beljonne, J. Am. Chem. Soc. 127, 4744 (2005).

${ }^{28}$ R. G. Kepler, V. S. Valencia, S. J. Jacobs, and J. J. McNamara, Synth. Met. 78, 227 (1996).

${ }^{29}$ I. B. Martini, A. D. Smith, and B. J. Schwartz, Phys. Rev. B 69, 035204 (2004).

${ }^{30}$ C. L. Gettinger, A. J. Heeger, J. M. Drake, and D. J. Pine, J. Chem. Phys. 101, 1673 (1994).

${ }^{31}$ S. J. Strickler and R. A. Berg, J. Chem. Phys. 37, 814 (1962).

${ }^{32}$ E. Peeters, A. M. Ramos, S. C. J. Meskers, and R. A. J. Janssen, J. Chem. Phys. 112, 9445 (2000).

${ }^{33}$ I. D. W. Samuel, G. Rumbles, C. J. Collison, R. H. Friend, S. C. Moratti, and A. B. Holmes, Synth. Met. 84, 497 (1997).

${ }^{34}$ J. H. Hsu, M. T. Hayashi, S. H. Lin, W. S. Fann, L. J. Rothberg, G. Y. Perng, and S. A. Chen, J. Phys. Chem. B 106, 8582 (2002).

${ }^{35}$ C. R. Sabanayagam, J. S. Eid, and A. Meller, Appl. Phys. Lett. 84, 1216 (2004).

${ }^{36}$ J. J. Liang, J. D. White, Y. C. Chen, C. F. Wang, J. C. Hsiang, T. S. Lim, W. Y. Sun, J. H. Hsu, C. P. Hsu, M. Hayashi, W. S. Fann, K. Y. Peng, and S. A. Chen, Phys. Rev. B 74, 085209 (2006).

${ }^{37}$ P. Kumar, A. Mehta, M. D. Dadmun, J. Zheng, L. Peyser, A. P. Bartko, R. M. Dickson, T. Thundat, B. G. Sumpter, D. W. Noid, and M. D. Barnes, J. Phys. Chem. B 107, 6252 (2003).

${ }^{38}$ K. Becker and J. M. Lupton, J. Am. Chem. Soc. 128, 6468 (2006).

${ }^{39}$ P. R. Hania, D. Thomsson, and I. G. Scheblykin, J. Phys. Chem. B 110, 25895 (2006).

${ }^{40}$ F. Schindler, J. M. Lupton, J. Muller, J. Feldmann, and U. Scherf, Nat. Mater. 5, 141 (2006).

${ }^{41}$ J. Yu, R. Lammi, A. J. Gesquiere, and P. F. Barbara, J. Phys. Chem. B 109, 10025 (2005).

${ }^{42}$ A. J. Gesquiere, S. J. Park, and P. F. Barbara, J. Am. Chem. Soc. 127, 9556 (2005). 